\title{
Péripéties genevoises de la psychiatrie légale fin-de-siècle
}

\author{
par Vincent Barras
}

\section{RÉSUMÉ}

Dans la deuxième moitié du XIX $X^{e}$ siècle se déroulent à Genève deux importants procès criminels. Les expertises médico-légales qui y ont lieu sont l'occasion et le prétexte de débats théoriques animés, débordant le cadre strictement local: des personnalités étrangères interviennent, des articles polémiques sont publiés dans les revues spécialisées françaises. On peut lire dans ces affrontements non seulement une lutte de pouvoir entre médecine et monde juridique, mais aussi les difficultés théoriques d'un domaine en pleine élaboration de ses propres concepts, qui trouveront une formulation stabilisée dans les théories freudiennes.

Deux procès criminels survenus à Genève pendant la deuxième moitié du $\mathrm{XIX}^{\mathrm{e}}$ siècle retiendront notre attention.

Le premier est celui de Marie Jeanneret, «âgée de 32 ans, garde-malade, née au Locle, demeurant à Genève, détenue, taille 4 pieds 7 pouces 2 lignes, corpulence ordinaire, cheveux et sourcils châtains, front découvert, yeux bruns, nez moyen, bouche moyenne, dents incomplètes, menton rond, teint pâle, visage ovale, démarche vive, signe particulier : cicatrices au dos et à la poitrine» ${ }^{1}$, et accusée d'avoir commis un attentat à la vie de neuf personnes en leur administrant des «substances pouvant donner la mort plus ou moins promptement». Le 26 novembre 1868, le jury à qui l'on a posé la question rituelle : «Marie Jeanneret est-elle coupable d'avoir volontairement attenté à la vie de ces neuf personnes?» répond: «Oui, avec circonstances atténuantes». La cour de justice criminelle considère que les faits déclarés constants par le jury constituent à l'égard de l'accusée le crime d'empoisonnement prévu par les art. 301 et 302 du Code Pénal et condamne l'accusée à la peine de 20 ans de travaux forcés ${ }^{2}$. La sécheresse de l'énoncé, ainsi consigné dans les règles par le greffe de la Cour de Justice Criminelle avec Jury, suffit mal à 
masquer une émotion qui surgit encore un siècle après. Tuer neuf personnes par empoisonnement, voilà qui n'est assurément pas une expérience quotidienne, fût-elle du magistrat le plus aguerri. Et fatalement, devant un crime aussi hors nature, surgit l'interrogation: qu'est-ce qui a bien pu pousser quelqu'un à un tel forfait? quelle est donc cette extraordinaire personnalité? Sans doute figurerait-elle en bonne place au palmarès des grands criminels de la littérature policière et de la presse à sensation, dans une catégorie particulière - dont la naissance accompagne curieusement celle de la profession même, et qui semble éveiller d'inquiétantes résonances dans l'imaginaire collectif de la fin du XIX ${ }^{\mathrm{e}}$ siècle (et sûrement du nôtre encore) -: l'«infirmière-empoisonneuse» ${ }^{3}$. La trajectoire de Marie Jeanneret telle que les minutes du procès nous permettent de la reconstituer est à cet égard exemplaire. Très tôt, elle démontre un goût affirmé pour la maladie sous toutes ses formes; après avoir suivi une école de gardes-malades à Lausanne, elle se lance dans une véritable tournée des médecins à travers la Suisse romande. Dans l'espace de quelques années, elle en consulte une vingtaine au moins, évoquant toute une série de symptômes, de la constipation opiniâtre à l'engorgement utérin en passant par la cécité brusque, exigeant les traitements les plus sévères, auxquels elle semble d'ailleurs se complaire, insistant même pour se faire cautériser au fer rouge, chez un médecin de Rolle, à l'occasion d'une mystérieuse «affection de l'épine dorsale» (ce dont témoignent ses cicatrices sur le dos et à la poitrine). Elle fait montre en ces années d'une attirance de plus en plus prononcée pour l'atropine, qu'elle s'administre à elle-même et aux malades dont elle a la charge dans diverses pensions de convalescence à Lausanne, Vevey, Genève, et dont neuf au moins, on l'a vu, décèdent. En juin 1868 seulement, après que plusieurs médecins genevois aient remarqué chez leurs patients, tous soignés par Marie Jeanneret, des symptômes communs et suspects: vomissements, malaises, symptômes cérébraux, éclate l'évidence. On découvre enfin le secret de la garde-malade: une pharmacie entière, essentiellement composée de drogues atropiniques, est celée dans son armoire.

Une accusée de cette trempe a de quoi soulever les passions, surtout dans le petit cercle de la médecine légale. Le procès de Marie Jeanneret est en effet le théâtre d'une bataille d'experts qui, comme souvent, nous en apprend probablement plus sur les protagonistes et sur le contexte théorique que sur l'accusée elle-même. Deux camps bien tranchés s'affrontent. Les docteurs Badan, médecin de la prison, Olivet, médecin directeur de l'Asile des Vernets et futur professeur de psychiatrie à la Faculté de Genève, ainsi que Duval, 
requis par le juge d'instruction d'examiner l'état mental de l'inculpée, établissent le rapport suivant:

«Marie Jeanneret est malade depuis plusieurs années; les symptômes multiples énumérés par elle se rapportent à un tempérament hystérique, dont les principales manifestations ont été, d'après son récit: Paralysie des extrémités et des sphincters. Crises nerveuses avec perte partielle ou complète de connaissance, pendant un temps plus ou moins long (quelques minutes ou quelques heures). Vomissements aqueux ou sanguinolents, revenant à peu près périodiquement, et liés à des désordres menstruels graves. Troubles de la vue, de la digestion, et de la sensibilité. La plupart de ces symptômes se sont également présentés depuis sa détention sauf la paralysie des extrémités. Un examen de près de deux heures ne nous permet de découvrir, chez la détenue, aucune anomalie de l'état mental; en particulier, elle n'a paru atteinte d'aucune hallucination pendant son séjour à la prison. Fait à Genève le 7 septembre 1868. Drs. Olivet, Badan, Duval.»

Pourtant, les médecins témoins - il n’en manque certes pas - qui avaient eu l'occasion d'examiner Marie Jeanneret émettent des opinions moins affirmatives, comme le docteur Lambert, de Lausanne:

«J'ai remarqué que Marie Jeanneret supportait avec courage les maux dont elle était atteinte, ainsi que les moyens destinés à les combattre, elle avait de la résolution et de l'entrain, mais je ne puis dire si par dessous se masquait une tendance vers l'hystérie.»

D’autres relèvent «le caractère fuyant», ou encore «l'esprit remuant et intrigant» de l'accusée. Chacun est troublé par son intelligence, sur laquelle viennent se greffer ces inexplicables crises de nerfs que, nous dit encore le docteur Goudet de Genève, «j'attribuais à sa nature historique [!]». On ne pourrait risquer meilleur calembour que ce lapsus calami pour dire combien le diagnostic d'hystérie est lié à l'époque où se déroule l'affaire; il n'en est alors point de plus adéquat pour recouvrir la zone incertaine de personnalité vers laquelle convergent, pour ainsi dire à leur insu, volonté d'intrigue, méchanceté perverse, mais aussi névropathie relevant peut-être d'un esprit aliéné, crises évoquant l'épilepsie et ses saisissements involontaires. Des profanes l'avaient eux aussi pressenti:

«De manière générale quant à son séjour à l'école», déclare A. Reymond, directeur de l'Ecole de gardes-malades de Lausanne, «elle a été régulière et convenable; j'ai bien remarqué quelque chose de singulier chez elle, mais elle accomplissait ses devoirs, faisait des visites lorsque je l'envoyais, comme les autres élèves. Cette singularité était quelque chose d'indéfinissable, consistant en une grande mobilité de caractère, quelque chose d'agité, causant beaucoup.»

Le doute s'insinue jusque dans l'esprit du procureur général, lequel, bien que fort sévère dans son acte d'accusation (il considère en effet que Marie 
Jeanneret est bel et bien coupable de neuf crimes d'empoisonnement), n'en reconnait pas moins que:

«il y a quelque chose de si extraordinaire dans des crimes commis en grand nombre, sans aucun but ou intérêt matériel appréciable, que l'idée d'un trouble dans les facultés mentales a dû se présenter la première à l'esprit des magistrats et des experts chargés de l'information. On est porté même à désirer pour l'honneur de l'humanité qu'il n'existe pas de perversité semblable à celle dont toute cette procédure offre le spectacle. Aussi l'un des premiers soins de Mr. le Juge d'instruction a-t-il été de faire examiner à ce point de vue la fille Jeanneret par trois docteurs. Mais après une sérieuse et longue investigation, ces docteurs ont déclaré dans leur rapport qu'ils ne pouvaient apercevoir chez elle aucune marque d'aliénation mentale. [...] Il faut cependant reconnaître que les docteurs vaudois qui l'ont soignée précédemment déclarent très nettement qu'ils l'ont toujours considérée comme hystérique et folâtre.»

Aussi peut-on raisonnablement penser que c'est grâce à cette particularité que le jury (qui, délibérant en secret, ne laisse hélas pas à la postérité de traces de la discussion aboutissant à la décision définitive) attribue à Marie Jeanneret les circonstances atténuantes. Mais le débat ne se termine pas avec la fin du procès. Le docteur Châtelain, médecin-adjoint à Préfargier et membre correspondant de la Société médico-psychologique de Paris, écrit peu après un long article consacré au procès dans l'organe de ce groupe professionnel si influent dans l'histoire de la psychiatrie française du XIX ${ }^{\mathrm{e}}$ siècle. N'ayant pu examiner personnellement la condamnée, et se fondant uniquement sur les pièces de la procédure, il se convainc néanmoins du grand intérêt scientifique de l'affaire. L'analyse est très longue et minutieuse: c'est qu'il s'agit, plus encore que de démontrer l'erreur, d'élever la démonstration à une véritable méthode, celle seule à laquelle l'expert médecin au fait des données nouvelles de la science devrait obéir. On recherchera les critères de l'aliénation dans trois ordres de faits. Les antécédents tout d'abord:

«Elle est, de l'aveu de tous, hystérique, nerveuse, impressionnable, remplie d'une activité agitée et tracassière; elle ne peut se fixer nulle part, mais change sans cesse de domicile; son caractère est haineux, envieux, aigri depuis l'enfance, son humeur passe subitement d'accès de tendresse et d'affection à des explosions de colère et à des flux d'invectives. En outre, elle est malade plus ou moins imaginaire, pousse la manie des traitements jusqu'à se complaire à des cautérisations douloureuses! [...] Enfin, nous devons ajouter qu'une bisaïeule de l'accusée était complètement aliénée pendant ses grossesses. [...] En somme, personne ne pourra contester que si cet ensemble de symptômes psychiques n'est pas encore l'aliénation, il n'en constitue du moins une puissante prédisposition. ${ }^{4}$

Les «circonstances particulières et nature des actes criminels» constituent le deuxième ordre de faits où l'expert doit puiser ses preuves: 
«La première chose qui frappe dans ces neufs empoisonnements commis par M. J. est l'absence de tout motif; elle n'a qu'un but: tuer pour tuer, ces trois mots résument tout; peu importe le sexe, l'âge, la position sociale, l'état de santé ou de maladie de sa victime; elle est irrésistiblement poussée à la faire périr. [...] C'est une machine à donner la mort et rien de plus.»

Et enfin, la «manière d'être de l'accusée après son emprisonnement et pendant les assises» est elle aussi une source précieuse d'indices:

«Le système de défense adopté par l'accusée est insoutenable et n'est dans aucun rapport, d'une part, avec le nombre et l'atrocité des faits incriminés, de l'autre, avec l'intelligence de celle qui les a commis; si nous osions nous servir de cette expression, nous dirions qu'il est ridicule.»

Voilà donc comment, selon Châtelain, il est possible - et nécessaire d'apprécier l'état mental d'un individu. La simple constatation de la présence ou de l'absence d'un symptôme d'aliénation - par exemple l'hallucination, symptôme alors à la mode chez les aliénistes et dont les trois experts avaient dûment constaté l'absence - ne suffit pas.

«Nous ne pouvons [...] nous contenter d'un examen de deux heures dans un cas pareil; ce sont des semaines, des mois d'observation qu'il aurait fallu ici.» En effet, «tout aliéniste sait qu'on peut être complètement aliéné sans que cette aliénation se traduise par des paroles extravagantes ou hors de propos; il y a un délire des actes, et le 〈fous tel que le public se le représente généralement est en somme un type assez rare. Tout, dans le cas particulier, ne concourt-il pas à prouver que Marie Jeanneret est atteinte de monomanie homicide au plus haut degré? Hystérie avec aberrations de la sensibilité, troubles des facultés affectives, folie morale, impulsions irrésistibles, actes insensés, hérédité: voilà bien de quoi bannir absolument la responsabilité.»

\section{II}

Les termes de cet insoluble débat: folie, avérée et indiscutable, dont la conséquence légale est l'absolution de toute responsabilité, ou crime, commis en plein possession des «facultés intellectuelles», et tombant sous le coup des mesures prévues par le Code Pénal ${ }^{5}$, se retrouvent de façon très étrangement similaire lors d'un procès qui a lieu près de vingt ans plus tard, en 1885 . On juge cette fois «la nommée Jeanne Emilie Deluermoz, femme de Joseph Lombardi, âgée de trente-trois ans, sans profession, genevoise, détenue» ${ }^{6}$, et accusée d'avoir «volontairement commis [trois] homicides sur la personne de [ses enfants] Pierre Eugène, Emile Elie, Joséphine Henriette Lombardi, en se servant contre [eux] d'un instrument tranchant, avec cette circonstance 
que ces homicides volontaires ont été commis avec préméditation, crimes prévus et punis par les articles 83, 249, 251 et 252 du Code Pénal», ainsi que «tenté de commettre un homicide volontaire sur son enfant Joseph Emile, en se servant contre lui d'un instrument tranchant, tentative manifestée par des actes extérieurs constituant un commencement d'exécution, et qui n'a été suspendue ou n'a manqué son effet que par des circonstances fortuites ou indépendantes de son auteur, avec cette circonstance que cette tentative d'homicide volontaire a été commise avec préméditation, tentative de crime prévue et punie par les articles 5, 83, 249, 251 et 252 du Code Pénal». A la question: «L'accusée est-elle coupable d'avoir volontairement commis trois homicides et tenté de commettre un homicide volontaire?», le jury répond cette fois sobrement: «Non», et en conséquence, Jeanne Emilie Deluermoz, femme Lombardi est acquittée de l'accusation de crime d'assassinat. C'est à nouveau, on s'en doute, un cas peu banal avec lequel la justice doit découdre : quelques mois à peine après le mariage de l'inculpée avec Joseph Lombardi commencent scènes d'ivrognerie, violentes menaces, soufflets et coups répétés, tant de la part de son mari que de sa belle-famille ${ }^{7}$. Peu à peu, nous dit-elle, s'installe dans son esprit le sombre dessein d'en finir avec cette vie cauchemardesque: le soir du 1 au 2 mai 1885, profitant d'une absence de son mari, infidèle de surcroît, elle égorge ses quatre enfants à l'aide d'un rasoir avant de tenter de s'empoisonner. Seul le dernier-né, âgé d'à peine trois ans et demi, survivra, ainsi qu'elle-même. A nouveau, on se croirait là en plein roman, un de ces romans populistes d'une absolue noirceur, dont le XIX ${ }^{\mathrm{e}}$ siècle finissant est si friand. Mais nous nous restreindrons une fois encore aux péripéties médico-légales complexes et bien réelles qu'entraîna le procès.

Le professeur Léon Revilliod, premier titulaire de la chaire de médecine à la Faculté nouvellement créée, examine en personne l'accusée, qui venait tout juste de reprendre connaissance après avoir commis ses crimes monstrueux, lors de son arrivée à l'Hôpital Cantonal:

\footnotetext{
«Sa physionomie exprime à première vue qu'elle n'a pas conscience de son état actuel et qu'elle doit être sous l'impression d'un délire tranquille. [...] Son regard se fixe par moments dans l'espace comme étant l'objet d'une hallucination. Elle dit en effet qu'elle voit des hommes, des cordes, etc. Le visage est un peu coloré, les pupilles dilatées ne réagissent pas à la lumière; les objets sont vus plus petits que nature. Elle accuse un peu de sécheresse de la gorge, de la soif, du mal de tête siégeant surtout au vertex. Le pouls est petit et rapide. Elle a son époque menstruelle. [Le lendemain], Mme L. retrouve sa raison, le souvenir des événements [...], son crime, ses hésitations, les liqueurs et l'atropine qu'elle a absorbés pour avoir la force de réaliser son projet. Elle raconte son crime en détail.»
} 
Aux yeux de Revilliod, qui fait montrer ici d'une surprenante culture psychiatrique, un tel délire ne peut être attribué à l'alcool, ni exclusivement à l'atropine : il s'agit bel et bien, chez une femme où se rencontre par ailleurs «une certaine prédisposition héréditaire ou acquise aux perversions de l'esprit», d'un accès maniaque. Le diagnostic se laisse en effet soupçonner à partir de toute une série d'indices retrouvés dans ses antécédents: maux de tête, visions obsessionnelles, rêves étranges, crises d'excitation nerveuse lors des époques menstruelles, et il se révèle dans toute son évidence lorsqu'on est attentif au déroulement de l'accès, qui

"présente tous les caractères de la manie homicide résumés par Maudsley ${ }^{8}$ : caractère paroxysmal de l'attentat, soulagement extraordinaire ressenti par le patient aussitôt après que l'acte a été accompli, fréquence avec laquelle l'attentat a pour victime un proche parent, indifférence du coupable après l'action quant à la nature épouvantable de l'acte.»

Emilie Lombardi, conclut-il, doit par conséquent être considérée comme irresponsable:

«Chez le criminel, il y a rapport direct et proportionnel entre le crime et l'intérêt, les passions qui sont en jeu. Dans ce cas, rien de semblable. Mme L. a tué ses enfants, possédée par une impulsion maladive irrésistible, malgré ou à cause de son amour maternel.»

Pourtant, à cette observation d'allure toute objective s'oppose le rapport du professeur de médecine légale, Hyppolite-Jean Gosse. Préoccupé lui aussi d'appliquer des critères rigoureux dans un domaine si délicat, il ne peut que constater qu'il n'y a chez l'accusée «aucun indice d'arrêt du développement», aucun de ces stigmates révélés chez les aliénés par la doctrine toute fraîche de la dégénérescence. De même, dans l'ascendance de l'accusée, qui fait elle aussi l'objet d'une enquête approfondie, on retrouve bien un grandoncle «atteint de folie systématique», ou encore un cousin germain «atteint de bégaiements». Mais l'expert médico-légiste ne constate «aucune maladie héréditaire ayant pu avoir une influence sur [sa] santé». Pas de troubles de l'intelligence non plus:

«Elle est peu lettrée, mais dans ce qu'elle écrit, il y a une certaine facilité, un langage imagé qui quelquefois n'est pas sans poésie. En revanche, elle possède une mémoire merveilleuse, que j'ai pris soin de vérifier à nombre de reprises et que je n'ai jamais trouvée en défaut. Elle m'a paru être intelligente.»

On pourrait bien sûr penser à un état de dépression ou de mélancolie, mais dans un tel cas, on retrouverait l'«évidence d'une douleur psychique nullement ou insuffisamment motivée extérieurement. [...] Cet état de souffrance 
morale générale et sans objet, cette dépression [n'ont] aucune raison d'être extérieure» prétend Gosse en se référant aux théories sur la mélancolie des aliénés du célèbre professeur autrichien von Krafft-Ebing ${ }^{9}$. Or ici, n’y a-t-il pas surabondance de motifs, de raisons extérieures : accumulation de ressentiments, haine progressivement développée, perspective de vie décourageante? Par conséquent, conclut-il,

\begin{abstract}
«la vie et les actes de Madame Lombardi sont ceux d'une personne qui a la pleine possession de ses facultés intellectuelles [...] Dans la série des actes qui se sont succédé, il n'y a aucun indice quelconque d'aliénation. [...] Il n'y a pas eu d'impulsion instinctive. [...] Elle a pris sa décision, elle est décidée à se tuer, mais elle ne se sent pas le courage de faire ce qu'elle croit son devoir, c'est-à-dire de tuer des enfants qu'elle adore, plutôt que de les laisser entre des mains qui, suivant elle, feraient leur malheur. Il lui faut du courage, elle en manque, elle prend de l'eau-de-vie et elle pleure, elle hésite, jusqu'au moment où l'alcool commence à agir. Ce fait seul nous montre que nous n'avons pas à faire à une aliénée. [...] Tout est raisonné, tout est logique; c'est de la violence raisonnable, c'est de la passion! c'est du désespoir! Ce n'est pas de la folie.»
\end{abstract}

Deux opinions aussi radicalement opposées ne peuvent que relancer le débat, qui se poursuit dans le tribunal tout d'abord, puis dans la presse spécialisée. Un trio d'experts est commis par le Juge d'Instruction: les docteurs Long, ancien médecin de la Métairie, près de Nyon, et Châtelain, déjà au monté au front lors de l'affaire précédente, et désormais retraité, penchent à l'instar de Revilliod, pour l'irresponsabilité, mais en soutenant la thèse d'une mélancolie sur laquelle s'est greffée «une véritable conception délirante». A leur long «rapport médico-légal de majorité» s'oppose tout aussi radicalement celui «de minorité» dû au docteur A. H. Vaucher, qui, en sa qualité de professeur d'accouchements à la Faculté de Genève, examine «l'état de santé de la femme Emilie Lombardi, [...] principalement au point de vue de l'activité sexuelle et les conséquences que l'on peut en tirer pour la rendre responsable ou l'absoudre du crime qu'elle a commis.» $O r$, là où ses collègues voient des symptômes caractéristiques de mélancolie, lui ne conclut qu'à une discrète anxiété qui «[s'explique] suffisamment par l'état pathologique de la prévenue, état résultant de ses souffrances morales, de ses fatigues physiques, de ses grossesses, du nourrissage». De plus, «n'y a-t-il pas dans ce crime pour mobile encore la vengeance contre la famille d'un mari qui l'a si cruellement éprouvée, afin de faire peser sur elle cette honte à laquelle les gens de son pays [la Haute-Savoie] attachent une si grande importance et dont son esprit a été imbus dès sa jeunesse?» Aussi n'hésite-t-il pas à déclarer «la femme Lombardi responsable du crime qu'elle a commis le ler mai 1885». Contre cet avis 
se dressent vigoureusement, outre pas moins de douze médecins entendus lors des sessions de la Cour (dont le fameux Jules Déjerine, d'origine genevoise et devenu professeur à la Faculté de Médecine de Paris), des voix plus lointaines, mais non moins prestigieuses, toutes conviées par l'avocat de la défense Adrien Lachenal à se joindre à ce concert de protestations. Après consultation des différents rapports de ceux qui ont effectivement examiné l'accusée, les docteurs Challand (directeur de l'Asile de Cery), Schaerer (directeur de la Clinique Waldau), Speyr (médecin adjoint à la Clinique Waldau), Fetscherin (directeur de l'Asile de St. Urban), le professeur von Krafft-Ebing lui-même, sans doute la plus grande autorité du moment en la matière, concordent tous : il ne s'agit certes pas d'une crise de manie, comme le pensait Revilliod, (car, comme dit Olivet, «il n’y a aucun mouvement inconscient ou impulsif»), mais bien d'un «état mélancolique, qui a longtemps caressé l'idée de destruction, et qui a amené la crise». Et KrafftEbing, pourfendant au passage l'interprétation trop restrictive de ses écrits par l'expert médico-légiste Gosse (qui aboutissait, on l'a vu, à des conclusions diamétralement opposées), conclut en traduisant cette commune conviction en des termes juridiques:

«Madame Lombardi est une aliénée. [...] Certainement elle n’a pas été responsable, n'étant pas libre d'esprit au moment du crime [...]. La réunion de la malheureuse mère à ses enfants, par la main du bourreau, serait peut-être un bienfait pour elle, mais dans tous les cas, ce serait un meurtre judiciaire, commis sur une aliénée digne de pitié.»

\section{III}

C'est l'Europe entière qui pourra prendre part a posteriori à ce procès; comme le précédent, il connaît les honneurs d'une publication dans un important journal ${ }^{10}$. Paul-Louis Ladame, neuro-psychiatre très actif installé à Genève, correspondant de multiples sociétés médicales suisses et étrangères ${ }^{11}$, y reprend l'essentiel du débat médico-légal en s'appliquant à démontrer la difficulté d'un tel cas:

«En dehors de cette folie bruyante, de cette manie aiguë, de ce délire hallucinatoire que tout le monde connaît et reconnaît immédiatement dès qu'il a éclaté, il existe d'autres formes d'aliénation mentale, plus profondes, plus insidieuses, qui ne peuvent être reconnues que par les aliénistes, et qui enlèvent de même complètement le libre arbitre du malade, bien qu'elles laissent intactes ses facultés intellectuelles, sa mémoire, sa raison et son jugement, apparemment du moins et d'habitude pendant une certaine période de la maladie seulement.» 
Est ainsi réactivé l'un des thèmes favoris et pour ainsi dire constitutifs de la psychiatrie naissante, la question de la compétence spéciale des aliénistes à l'intérieur de la profession médicale, et plus largement, de celle-ci par rapport à d'autres professions en matière d'aliénation mentale. La médecine avait beau se sentir forte d'une tradition millénaire, les impulsions nouvelles, au tournant du XVIII ${ }^{\mathrm{e}}$ siècle, ne furent pas seulement le fait de médecins. Le citoyen Pussin à Bicêtre, le pasteur quaker William Tuke à York, pour ne citer que deux exemples fondamentaux de l'histoire de la psychiatrie, n'avaient aucune formation médicale, et furent pourtant des acteurs capitaux de ce qu'on peut appeler la naissance de la psychiatrie moderne. Mais cette question, on s'en doute, fut particulièrement cruciale dans le secteur de la "psychiatrie légale» (bien que ce terme n'existât pas encore en tant que tel). Ainsi, dans les années 1820, à la suite de la publication d'un des premiers ouvrages qui traitât de ces matières ${ }^{12}$, éclatait en France une polémique dont le retentissement fut énorme au sein des revues spécialisées, mais aussi dans la presse générale. On pouvait lire par exemple:

«Si la loi veut que les médecins soient consultés sur la folie, c'est sans doute par respect pour l'usage, et rien ne serait plus gratuit que la présomption de la capacité spéciale des médecins en pareille matière. De bonne foi, il n'est aucun homme d'un jugement aussi sain qui n'y soit aussi compétent que M. Pinel ou M. Esquirol et qui n'ait encore sur eux l'avantage d'être étranger à toute prévention scientifique..$^{1{ }^{3}}$,

En effet, la folie présente-t-elle des symptômes si particuliers que seul le médecin puisse reconnaître?

«C'est seulement dans ce cas, déclare un avocat à la Cour Royale de Paris, que sa présence serait nécessaire dans les tribunaux. Quand la folie est évidente, on n'a pas besoin d'un expert; le médecin ne serait donc utile qu'autant qu'il y aurait doute, et qu'il parviendrait à le dissiper. ${ }^{14} \gg$

Or, on compte deux ordres de symptômes de la folie, ceux de l'intelligence et de la pensée (ce qui constitue le délire) et ceux survenant dans les fonctions organiques; et seuls les derniers sont du domaine de la médecine. Encore faudrait-il, selon les détracteurs de l'expertise médicale en matière d'aliénation mentale, que ces symptômes soient caractéristiques de la folie. Mais cela est loin d'être le cas: «Souvent même ils ne l'accompagnent pas, et leur absence n'empêche pas qu'elle existe. ${ }^{15}$ » Cette «anti-psychiatrie» avant la lettre n'est donc pas simplement le fait de juristes acharnés à défendre une portion de leur pouvoir. Dès le départ, on n'est pas prêt à recevoir comme telle la nouvelle spécialité au sein même de la profession. Difficile en effet 
d'admettre sans plus l'existence de certaines formes d'aliénation mentale proposées par le camp opposé, celui des aliénistes, qui revendiquent la nécessité d'une connaissance spécialisée. C'est bien sûr autour de la monomanie que se cristallisent les débats ${ }^{16}$. Etienne Esquirol avait proposé le terme dès 1810 environ, afin de remplacer le terme ambigu selon lui de mélancolie; avec quelques-uns de ses élèves, il avait très vite attiré l'attention sur les aspects médico-légaux très particuliers de certaines des variétés de cette entité:

«Certains aliénés tuent, motivent leur affreuse détermination, raisonnent leurs actions, et ont la conscience du mal qu'ils commettent. [...] Ils sont irrésistiblement portés à se déchirer, à se détruire, à tuer leurs semblables. ${ }^{17}$ »

Le propre des monomaniaques homicides, dans cette première phase d'élaboration théorique, est que leur aliénation ne se manifeste en fin de compte qu'à travers un seul symptôme: l'acte criminel justement. Sinon, tout désordre intellectuel ou affectif est absent. On comprend comment, suivant cette logique, a pu fleurir dans les diverses classifications des maladies mentales proposées le long du siècle un rameau spécifique le long duquel chaque acte criminel pouvait qualifier une nouvelle entité morbide: kleptomanie, pyromanie, érotomanie, morphinisme, impulsions pathologiques à la fuite. Mais on comprend aussi la difficulté qu'éprouveront longtemps juristes et médecins à entériner les théories des aliénistes. Comment concevoir la possibilité d'une folie si extraordinairement intense, et pourtant invisible jusqu'à ce qu'elle se manifeste à travers un crime atroce? Comment le médecin, tout spécialiste qu'il soit, pourrait dès lors en rendre compte, la prévoir, espérer peut-être exercer quelque pouvoir sur cette forme si spéciale, dont le propre est justement de se dérober? A cette véritable aporie, les aliénistes ne peuvent guère qu'opposer tautologiquement la force de leur «habitude», de leur «métier»:

\begin{abstract}
«Nous ignorons beaucoup de faits, c'est vrai; mais est-ce à dire pour cela que les gens du monde n'en ignorent pas cent fois davantage? [...] A force de vivre [dans l'obscurité], et d'exercer nos sens à nous y reconnaitre, nous nous sommes fait, pour ainsi dire, une sorte de faculté visuelle, toute artificielle et toute personnelle, il est vrai que nous sommes parvenus à nous y tenir tant bien que mal, à voir quelque chose là où les autres ne sauraient rien voir, et sauf les faux pas, qui sont inévitables dans les endroits où il fait par trop noir, nous savons fort bien nous tirer d'affaire. ${ }^{18}{ }^{\prime}$
\end{abstract}

Et soixante ans plus tard, c'est le même argument à peine transposé que reprend Ladame: il existe des formes d'aliénation mentale insidieuses et 
profondes, que les aliénistes seuls peuvent reconnaître. D'où la volonté, mais qui est aussi une nécessité théorique, de s'imposer sur un terrain délicat, que les juristes revendiquent comme propriété ancestrale. L'examen du crime en tant que tel, des motifs éventuels qui l'ont suscité, est susceptible de procurer à l'œil exercé une foule d'indices. Châtelain lui aussi ne s'y trompe pas, qui base l'essentiel de son argumentation sur les «circonstances particulières et nature des actes incriminés» de Marie Jeanneret. Chez elle, on constate certes un «ensemble de symptômes psychiques» bien particuliers que l'on retrouve lorsqu'on se penche sur ses «antécédents», mais cette «puissante prédisposition» n'est pas l'aliénation. C'est bien le crime et son contexte qui fournissent la preuve:

«En effet, il n'y a que deux mobiles possibles dans le crime: la passion avec tous ses écarts, et la folie avec toutes ses extravagances; mais le crime commis par la passion a des motifs, un but, il est conséquent, logique avec lui-même et relativement raisonnable, tandis que la folie n'a le plus souvent ni but ni motifs raisonnables pour le crime qui devient dès lors illogique, inconséquent, absurde.»

Or, à y regarder de près,

«l'accusée ne se cache pas, elle agit ouvertement au risque de se trahir; machinalement, instinctivement, elle dit tout haut ce qu'elle pense tout bas, puis tout à coup survient, comme un revirement de l'impulsion fatale, un cri suprême de la raison et de la conscience qui semblent vouloir surgir de nouveau du milieu de cet abîme de délire.»

C'est précisement ce qu'un magistrat, et même un médecin en apparence aussi qualifié qu'Olivet, ne saura déceler, le propre de cette forme si spéciale d'aliénation, ce «délire des actes», qui signe ce que Châtelain étiquette, faute de mieux (et l'on peut penser que c'est une des dernières occurrences du diagnostic dans l'histoire de la psychiatrie légale), comme une monomanie homicide ${ }^{19}$. Aussi porte-t-il la critique sur l'examen par trop sommaire des trois experts commis par le juge d'instruction. Le simple sens clinique, voire l'examen scrupuleux des symptômes concrets, visibles, ne suffit plus. C'est l'acte lui-même et ses «circonstances» qu'il faut convoquer pour démontrer l'existence de ce qui est l'évidence pour Châtelain, mais qui fait figure de simple postulat théorique pour les médecins adversaires de cette doctrine: «cette impulsion irrésistible et jusqu'à un certain point inconsciente». De même, lors du procès d'Emilie Lombardi, Gosse, médecin-légiste confirmé, a beau faire appel à des méthodes tout à fait modernes; l'établissement d'un arbre généalogique le plus étendu possible, la recherche des stigmates caractéristiques d'une dégénérescence. Nous pouvons repérer ici une application 
précoce des théories de l'Ecole italienne d'Anthropologie Criminelle, qui fera beaucoup parler d'elle et influencera durablement la criminologie. Et pourtant lui non plus n'a pas su voir «ces formes d'aliénation mentale plus profondes, plus insidieuses, qui ne peuvent être reconnues que par les aliénistes et qui enlèvent de même complètement le libre arbitre du malade», ces états pathologiques mentaux que Ladame considère comme «l'un des symptômes les plus fréquents des états de dépression psychique dont le type est la mélancolie sous toutes ses formes». Ce dernier a ensuite beau jeu de développer, payant à son tour son écot à l'esprit positiviste régnant, une analogie des processus mentaux avec les phénomènes neurologiques fraîchement mis en lumière:

\begin{abstract}
«On ne s'expliquerait pas l'horreur des crimes commis avec sang-froid par les aliénés de cette espèce si l'on ne savait pas qu'ils sont atteints d'anesthésie psychique. Un malade doit subir une opération douloureuse, on l'endort par l'éther ou le chloroforme qui lui enlèvent complètement le sentiment de la douleur. [...] Or il existe certaines maladies nerveuses qui produisent les mêmes effets sur différentes parties du corps; ce sont les anesthésies. [...] En pathologie mentale, nous trouvons un symptôme analogue; c'est lorsque le malade ne perçoit plus normalement la douleur morale, lorsqu'il existe une obtusion des sentiments affectifs.»
\end{abstract}

Quelque cent ans plus tard, ces controverses peuvent paraître d'importance somme toute mineure. On peut s'interroger sur leur influence effective lors de chacun de ces deux procès. Faut-il parler dans un cas d'un jury qui, à l'image des médecins experts, serait resté plus ou moins imperméable aux idées nouvelles? Peut-on voir dans l'autre le triomphe d'une opinion médicale éclairée ayant conquis, face à l'obscurantisme adverse, l'opinion juridique? Marie Jeanneret, qui fut jugée coupable avec circonstances atténuantes et passera vingt années en pénitencier, fut-elle victime d'une «erreur judiciaire», à l'inverse d'Emilie Lombardi, jugée non coupable, acquittée, et terminant ses jours internée à l'Asile des Vernets? Aujourd'hui, à l'heure où psychiatres et médecins s'interrogent de concert sur la pertinence de la notion d'irresponsabilité pénale des malades mentaux ${ }^{20}$, il semble difficile et peut-être vain de vouloir répondre à de telles questions, qui bien inévitablement surgissent, et qui, appliquées à des situations actuelles, n'entraîneraient sans aucun doute pas de réponse claire et univoque. L'intérêt historique des documents d'archive judiciaire ici brièvement présentés est 
ailleurs ${ }^{21}$ : il permet d'apprécier la vision vertigineuse qui s'ouvrit aux médecins, confrontés au monde privé, à la vie intime des petites gens à Genève au $\mathrm{XIX}^{\mathrm{e}}$ siècle. S'impliquer ainsi dans le quotidien, acquérir peu à peu un pouvoir sans précédent sur les manifestations objectives de la maladie, sur les corps souffrants, c'est aussi, pour eux, et peut-être sans qu'ils en aient pleinement conscience, voir se dégager les espaces immatériels et insondables de la subjectivité, dont la prise en compte ne s'est pas faite sans peines ni controverses théoriques et pratiques. Le conflit des compétences qui ressort ici avec une particulière netteté en fut une des modalités. Il s'inscrit dans une problématique du visible et du non-visible dans l'homme, de ce qu'en lui on peut saisir et de ce qui risque d'échapper indéfiniment à toute prise, qui travailla en profondeur toute la médecine de ce siècle. Les vies tragiques de Marie Jeanneret et d'Emilie Lombardi, ainsi perçues par le regard médico-légiste de leur temps, furent d'une certaine manière le difficile mais privilégié laboratoire d'essais duquel émana, magistralement dénouée au tournant du siècle, quelque chose comme l'idée d'inconscient. 
1 Comme cette première citation, l'ensemble de celles qui concernent le procès de Marie Jeanneret est tiré des Archives d'Etat de Genève (AEG), Jur. Pen. X n 578, 1868, et Jur. Pen. W, 3, no 222 .

2 Le jury n'eût-il pas accordé les circonstances atténuantes, le châtiment eût alors très certainement été la peine de mort.

3 Autre exemple, qui défrayait récemment la chronique internationale : dans les années 1980 , une infirmière se rendait coupable du meurtre par empoisonnement de 14 pensionnaires d'une clinique gériatrique autrichienne.

4 Cette citation, de même que les suivantes du Dr. Châtelain, est tirée de son article: Considérations médico-légales sur l'état mental de la nommée Marie Jeanneret, convaincue d'avoir commis neuf empoisonnements, in Annales médico-psychologiques 5érie, 1, 1869, pp. 248-274.

5 L'article 52 du Code Pénal du Canton de Genève de 1874 reprend les principes de l'article 64 du Code Pénal français de 1810, (qui, pour l'essentiel, régissait jusque-là le Canton de Genève: C. Flammer, A., Lois pénales d'instruction criminelle et de police qui forment en ces matières, avec les codes français et le code pénal militaire, la législation du Canton de Genève, Genève, 1962); il est ainsi libellé: «Il n'y a pas d'infraction lorsque l'accusé ou le prévenu était en état d'aliénation mentale au moment où le fait incriminé a eu lieu ou s'il a été contraint par une force à laquelle il n'a pas pu résister.»

6 Cette citation, ainsi que celles qui se rapportent au procès Lombardi, est tirée des AEG, Jur. Pen. X, nº 737, 1885, et Jur. Pen. W, 4, nº 55 .

7 La biographie contenue dans les pièces du procès et rédigée sur cinq cahiers de la main même de la prévenue lors de son séjour à la prison de Saint-Antoine avant le procès constitue un extraordinaire document d'histoire sociale: elle nous conte dans le menu son enfance en Haute-Savoie, comment, délaissée par un père ivrogne après le décès prématuré de sa mère, elle est élevée par des oncles brutaux et livrés à la boisson, puis comment, arrivée à Genève pour travailler, elle se retrouve une fois de plus battue comme plâtre par son logeur, avant de trouver un mari, buveur lui aussi, qui décède peu après. Suit son deuxième mariage avec J. Lombardi...

8 Henry Maudsley (1835-1918): psychiatre anglais réputé, auteur notamment de Physiologie et pathologie de l'esprit en 1867 et de Le crime et la folie, dont une traduction française paraît en 1874 .

9 Richard Freiherr Baron von Krafft-Ebing (1840-1902): élève de Griesinger à Zürich, il enseigne ensuite la psychiatrie en Allemagne et en Autriche. Il devient mondialement connu pour ses ouvrages des psychiatrie légale (dont La responsabilité criminelle et la capacité civile dans les états de trouble intellectuel, ouvrage traduit en français en 1875 par le psychiatre neuchâtelois Châtelain, qui intervient dans les deux procès présentés ici) et sa fameuse Psychopathia Sexualis, datant de 1886, première enquête exhaustive sur les anomalies sexuelles, à laquelle Freud ne cessera de se référer.

10 Ladame Paul-Louis, Relation de l'affaire Lombardi. (Suicide combiné d'assassinats commis par une mère sur ses enfants), in Archives de l'Anthropologie Criminelle et des Sciences Pénales 1, 1886, pp.436-460, et 2, 1887, pp.52-85.

11 Sa carrière et ses intérêts couvrent un très large champ, qui s'étend de la clinique et de la 
pathologie neurologique, en passant par la psychiatrie, la médecine légale, l'hygiène publique, jusqu'à des problèmes d'histoire de la médecine. Une telle figure, typique d'une certaine manière de concevoir et pratiquer la médecine dans la deuxième moitié du XIX ${ }^{\mathrm{e}}$ siècle, mériterait une étude spécifique. Pour un aperçu, cf. Naville F., Notice nécrologique sur le Docteur Paul-Louis Ladame, in Bulletin de l'Institut National Genevois 45, 1922-23, pp.91-122 (qui comprend également une liste des publications de Ladame).

12 Georget Etienne, Examen médical des procès criminels des nommés Léger, Feldtmann, Lecouffe, Jean-Pierre et Papavoine, dans lesquels l'aliénation mentale a été alléguée comme moyen de défense, suivi de quelques considérations médico-légales sur la liberté morale, Paris, 1825.

13 Coste, Urbain, Compte-rendu du Dictionnaire abrégé des Sciences Médicales, t. XI, XII et XIII, in Journal Universel des Sciences Médicales 43, juillet 1826, pp.47-69.

14 Regnault, Elias, Du degré de compétence des médecins dans les questions judiciaires relatives aux aliénations mentales, et des théories physiologiques sur la monomanie, Paris, 1828, pp.45.

15 ibid., p.7.

16 Cf. Goldstein, Jan, Console and Classify. The French Psychiatric Profession in the Nineteenth Century, Cambridge, 1987, en particulier le chapitre V: «Monomania», pp. 152-196.

17 Esquirol, Etienne, Des maladies mentales considérées sous les rapports médical, hygiénique et médico-légal, Bruxelles, 1838, t. I, p. 376 (Chap.11: «De la monomanie»).

18 Royer-Collard, Hippolyte, Compte rendu de l'ouvrage de E. Regnault, in Journal Hebdomadaire de Médecine 2, 1829, pp.181-204.

19 En effet, la monomanie comme entité nosologique (et la monomanie homicide en particulier) fut d'ailleurs difficilement admise par les psychiatres anglo-saxons ou allemands, et soumise en France, depuis les années 1840 à une critique serrée: on en nie son caractère d'espèce, préférant inscrire ces étranges «impulsions irrésistibles» qui caractérisent le monomane dans des catégories plus larges: folie morale, folie lucide, manie de caractère, dégénérés, etc. Cf. notamment Falret Jean-Pierre, Des maladies mentales et des asiles d'aliénés, Paris, 1864, (chap. V: «De la non-existence de la monomanie», publié initialement en 1854 dans les Archives Générales de Médecine). Châtelain, psychiatre à la page, est conscient de la difficulté: «Nous n'ignorons pas que ce mot est en grande partie hors de cours, mais comment, sans lui, dénommer le cas spécial ?» ajoute-t-il en note.

20 Cf. par exemple Poncela Pierrette, Le droit pénal en folie ou l'impossible vérité, in Revue de science criminelle et de droit comparé 1, 1986, pp. 61-68.

21 Signalons deux ouvrages récemment parus s'intéressant à l'histoire de l'expertise médicolégale, respectivement en France et en Ecosse: Harris Ruth, Murders and Madness, Medecine, Law, and Society in the fin de siècle, Oxford, 1989, et Crowther, Anne M \& White, Brenda, On Soul and Conscience. The Medical Expert and Crime, Aberdeen, 1988. 


\section{Summary}

Vicissitudes in Geneva forensic psychiatry towards the end of the 19th century

In the second half of the 19th century two sensational criminal trials took place at Geneva. They lead to theoretical debates about insanity and responsibility on an international level, which reveal not only a contest for power between medicine and law; they also show us a science in making in search for reliable concepts. Later it will find them in Freud's theories.

\section{Zusammenfassung}

\section{Umdenken in der Genfer Gerichtspsychiatrie gegen Ende des 19. Jahrhunderts}

In der 2. Hälfte des 19. Jahrhunderts finden in Genf zwei aufsehenerregende Kriminalprozesse statt. Sie geben Anlass zu grundsätzlichen Auseinandersetzungen über Geisteskrankheit und Schuldfähigkeit, an welchen sich auch ausländische Persönlichkeiten beteiligen. Es geht dabei nicht nur um einen Machtkampf zwischen Medizin und Jurisprudenz; eine in voller Entwicklung begriffene Wissenschaft sucht vielmehr nach verlässlichen Grundvorstellungen. Später wird sie diese mit Hilfe der Freudschen Theorien finden.

Dr. Vincent Barras

Institut Louis Jeantet d'histoire de la médecine Centre médical universitaire

1211 Genève 4 